\title{
A Data Analysis platform for high altitude bio monitoring using DEVOPS principles and time series machine learn- ing
}

\author{
Felix Constantin Adochiei ${ }^{1 *}$, Radu Ciucu ${ }^{2}$, Ioana Raluca Adochiei ${ }^{3}$, Florin Ciprian Argatu ${ }^{4}$, Ciprian- \\ Marius Larco ${ }^{5}$, Miron Casian ${ }^{6}$ \\ 1, 2,4 Faculty of Electrical Engineering, University Politehnica of Bucharest, Bucharest, Romania \\ 3, 5 Department of Aircraft Integrated Systems and Mechanics, Military Technical Academy "Ferdinand I" \\ Bucharest, Bucharest, Romania \\ ${ }^{6}$ Faculty of Automatic Control and Computer Engineering," Gheorghe Asachi" Te chnical University of Iasi, Iași, Romania
}

\section{Keywords}

Magnetic Resonance-

Imaging (MRI)

Digital Video Recorder (DVR)

Devops

Biomonitoring

Long-short term memory

Received: 4 December 2018

Accepted: 9 January 2019

Published: 20 February 2019

\begin{abstract}
This paper proposes a software platform for correlating re-al-time physiological data using microservices and time series analysis with machine learning. We use a devops type architecture with multiple containers governing specific aspects of our platform. Several micro-services had been designed to orchestrate and process multiple data streams in real time to run the analytics and correlation protocol. The data ingested by our platform is provided in an intuitive data visualization interface that binds together digital elevation models and geospatial data with the blood oxygen saturation and heart rate of a subject. The platform provides an accurate representation of a subject's physical performance in high altitude/low oxygen environments.
\end{abstract}

\section{INTRODUCTION}

The effects of high-altitude environments range from reduced oxygen to a lower atmospheric pressure. Such effects can inflict hypoxia and have a direct impact on the cardiovascular system $[1,2,3,4,5,6]$.

Electrocardiogram is one of the most common evaluation techniques and offers a wide range of information about a subject's state. It offers insights about chemical imbalances and electrophysiological conditions. Among the most common conditions are arrhythmias and $\operatorname{HRV}[3,4,5,7]$.

HRV deals with the variations of the time intervals be-tween the heart beats. It usually precedes a myocardia infarct. Various techniques are used for heart rate analysis, such as Ballistocardiograph (BCG), Photopletismography (PPG) and electrocardiogram, which is considered to be the most reliable since it allows the assement of the full wave-form $[1,8,9]$. DEVOPS is an IEEE standard and a software design philosophy recently popularized for making web platforms highly scalable, extremely easy to automate and capable of continuous integration and improvement. The devops field is directly tied to cloud computing and artificial intelligence as the need for scalable and fast solutions is on the rise. We developed our platform using the latest and most scalable solutions on the open-source scene [10,11].

By using data fusion procedures, satellite data can be three dimensionally reconstructed and directly rendered in the

\footnotetext{
${ }^{*}$ Corresponding author: Felix Constantin Adochiei

†email: felix.adochiei@upb.ro
} 
browser window using HTML5 and WebGL 2.0 meth-ods. Currently, wearable technologies are profiting of a raised interest from both the consumers and companies. One of the most recent trends which was theorized in 2005-2006 is the idea of a Wireless Body Area Network (WBAN) system that allows multiple intelligent sensors to relate to various wireless technologies, such as Bluetooth and Bluetooth Low Energy (BLE), Wi-Fi or ZigBee $[12,13]$.

By connecting multiple sensors to a mobile terminal, parameters such as oxygen saturation, heart-rate, temperature, body position and ECG could be measured, stored or sent to a server $[14,15,16,17]$.

In this paper, we proposed a platform dedicated to recording ECG, HR and SpO2 using a WBAN application for Android devices as well as tracking using GPS and BARO altitude data from the mobile terminal for real-time positioning on a 3D model of a mountain.

In the Materials and Methods section, we will describe the data types used for the tracking application, the correlation between the altitude and GPS data, the topography files used in the 3D reconstruction of the mountain area and the methods we used for the representation of HR and ECG and SpO2 on the 3D map $[18,19]$.

The results section will showcase each platform component. We will describe the devops development cycle and emphasize on the scalability of our solution. Furthermore, we will describe each platform component, from the frontend interface that displays real time data to the backend services which store and communicate the data through the API. Lastly, we will present our scalable solution for LSTM pattern recognition service. We will conclude the paper by discussing the perfor-mance of the platform, the devops philosophy impact over the development cycle. Moreover, we will sketch planed features based on our experience with the platform $[19,20]$.

\section{METHODS}

\section{A. Devops}

Devops is an IEEE standard that deals with the organizational and developmental processes of software platforms and automation frameworks. It is an acronym that stands for Development Operations, and it threats all aspects of itself as services and micoservices. A virtualization pipeline is usually implemented to orchestrate, schedule and deploy software platforms.

Devops is well suited for data ingestion and data processing in real time by employing persistent memory databases and fast data schemas. Each unit can be replicated and then purged from the memory while continuous deliv- ering real time support $[1,21,22]$.

\section{B. Digital Elevation Models}

Digital elevation models are either two dimensional ar-rays or sets of irregular networks of points.

NASA provides multiple sets of data, such as SRTM and ASTER, which are then translated into 3D displacement maps in order to achieve a realistic model of the Earth [23]. Shuttle Radar Topographic Mission (SRTM) DEM data sets are obtained by satellites with radar technologies, which makes the results accurate as the atmosphere conditions do not affect electromagnetic waves and their ability to bounce back to the receiver on the satellite. SRTM data as of 2006 is $90 \%$ accurate with errors of around 16 meters outside of the US and 9 meters for the North American data sets.

Since 2016, DEM NASA data sets can be obtained freely from the United States Geological Survey online platform. The data sets consist of two-dimensional greyscale images, with luminosity representing the elevation levels (Figure 1).

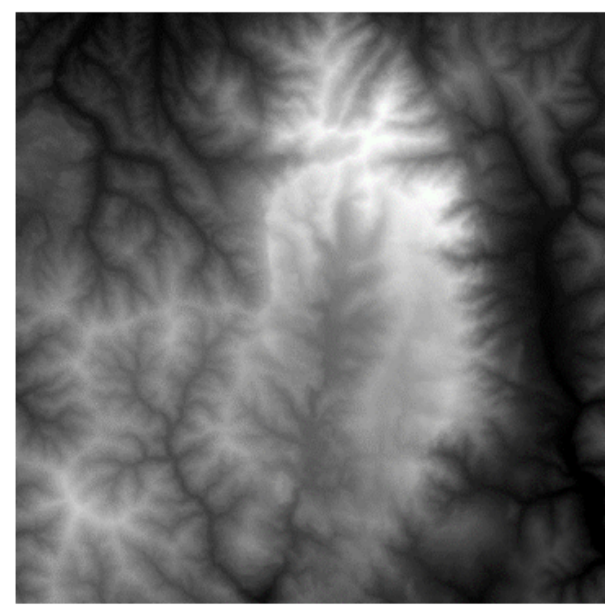

Fig. 1. Displacement map of bucegi massive

\section{Long-Short Term Memory (LSTM)}

LSTM neural networks are a type of recurrent neural networks specialized in time series prediction and pattern classification. Time series data is characterized by time step units and the periodic nature of the signal or the dataset. LSTM allows for specific parame-ters to become dynamically available as neuronal weights during use.

\section{Hardware Materials}

The acquisition equipment is designed around a TI microcontroller MSP430, with a frequency of $16 \mathrm{MHZ}$ and a resolution of 16 bits. The data transmission was realized by using the Bluetooth system on a chip model the Anaren A2541. The module features low power consumption of around $14 \mathrm{~mA}[4,15,24]$. 
ISSN: $2414-4592$

DOI: $10.20474 /$ jater-5.1.2 
The ECG Signal acquisition was done using an analog ECG interface from TI ADS1293. The chip features 3 dedi-cated channels with a sampling rate of $25.6 \mathrm{ksps}$, and is connected to the microcontroller trough an SPI interface.

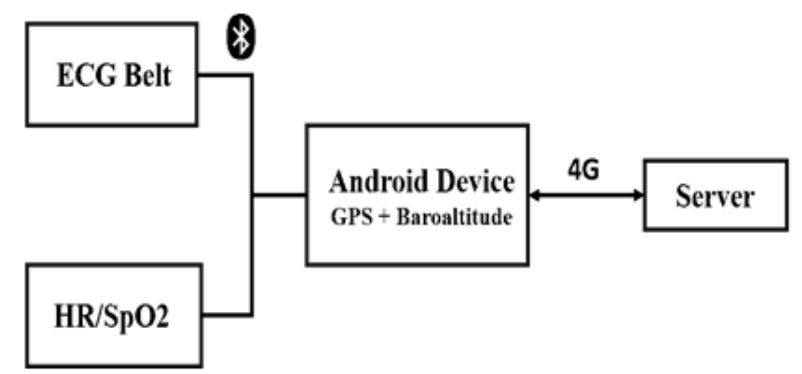

Fig. 2. Block diagram

The wearable device is strapped to the chest of the subject by using a belt. Moreover, the electrodes were fabricat- ed from a flexible conductor to ensure an adequate contact while the subject is moving (Figure 3 ).

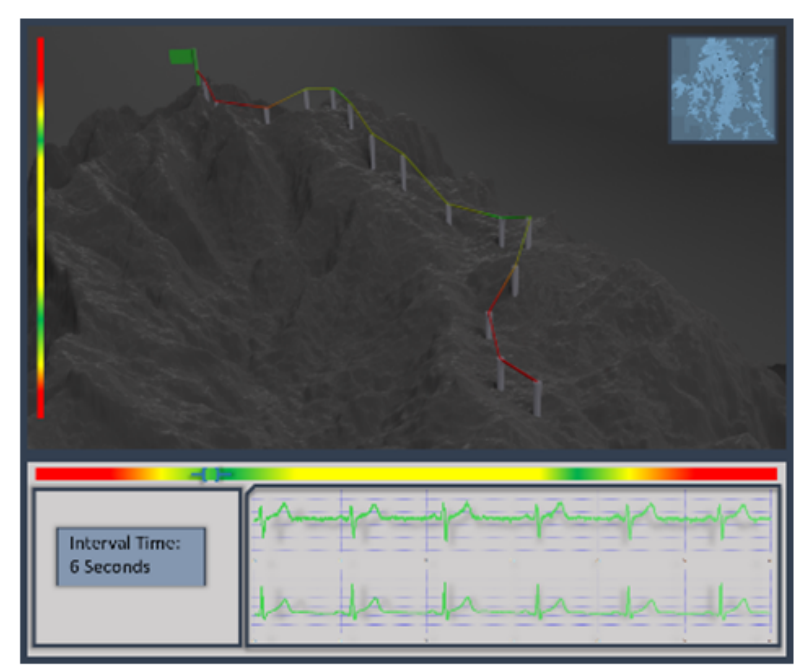

Fig. 3. Graphical user interface

\section{RESULTS}

We chose to implement our platform using a DEVOPS approach. The brickwork consists of docker container which allow for fast replication, communication and routing. The main reason behind the use of Linux containers is scalability. We can allocate a container for each subject of a study session.

The two main paradigms of our platform are the backend architecture and the frontend interface. Our backend is the support pillar for the storage, processing and data input features while the frontend allows for a detailed overview of the geographical and biological data.

\section{A. Backend Services and Microservices}

Our backend architecture is comprised of two main services:

A data ingestion service for collecting and storing real time data and a data processing service for time series classification.

The core of our services are linux containers built using Docker. The services contain all the software components needed to run a a specific task. All services communicate using a JavaScript API that returns JSON formatted da-ta $[24,25,26,27]$.

\section{B. Data Ingestion Service}

We developed a Linux container with main purpose of directly connecting over the internet using a WebSocket type connection to the mobile device to establish a continuous data stream. Our WebSocket server was built using Node.js with the Express web framework. This service has a database connector in order to send each sample coming from the subject. 
Our database schema was implemented with a time series structure using the document-oriented database MongoDB and a caching service called Redis. We chose Redis as a fast caching service for our machine learning and pattern classification components. Redis can store value-keys using persistent memory or RAM for fast access and a distributed approach.

Our Web Socket connection receives time series objects consisting of JSON formatted text. This data corresponds to our database schema and is identified by using a timestamp coming from the mobile device.

\section{Data Processing Service}

We implemented our pattern recognition service using a LSTM model. Our pattern recognition component was built using python and containerized with a special version of Docker for GPU access. The data ingested by our first service is made available trough an API that provides a specific number of samples. The time step of our model is adjustable using a frontend API.

\section{Frontend Interface}

Our frontend interface provides a realistic overview of the monitoring conditions and the GPS tracked route.
The digital elevation model is implemented using a merged height map of a specific region applied onto a plane. The displaced model represents the geographical shapes in three dimensions. We developed the data visuali-zation using a WebGL framework called three js.

Our interface holds two key features:

- Geographical overview

- Biological Monitoring

The geographical overview consists of Geolocation data fusioned together using three dimensional markers with our geographical displacement models. The geolocation data is directly extracted from the Android terminal using system routines (Android System API) and is represented by the altitude, speed, latitude and longitude.

In order to obtain a 3D model, we generated a displacement map by applying the DEM data to a two-dimensional plane resulting in a tridimensional mesh, in which vertices are displaced based on the intensity of the values contained in the data file. The displacement of the mesh was done along the $\mathrm{z}$ axis and was treated as a linear information since it is not con-sidered an RGB image, nor the contents representing the coloring of the terrain but the elevation levels. The resulted 3D model can be viewed in Figure 4.
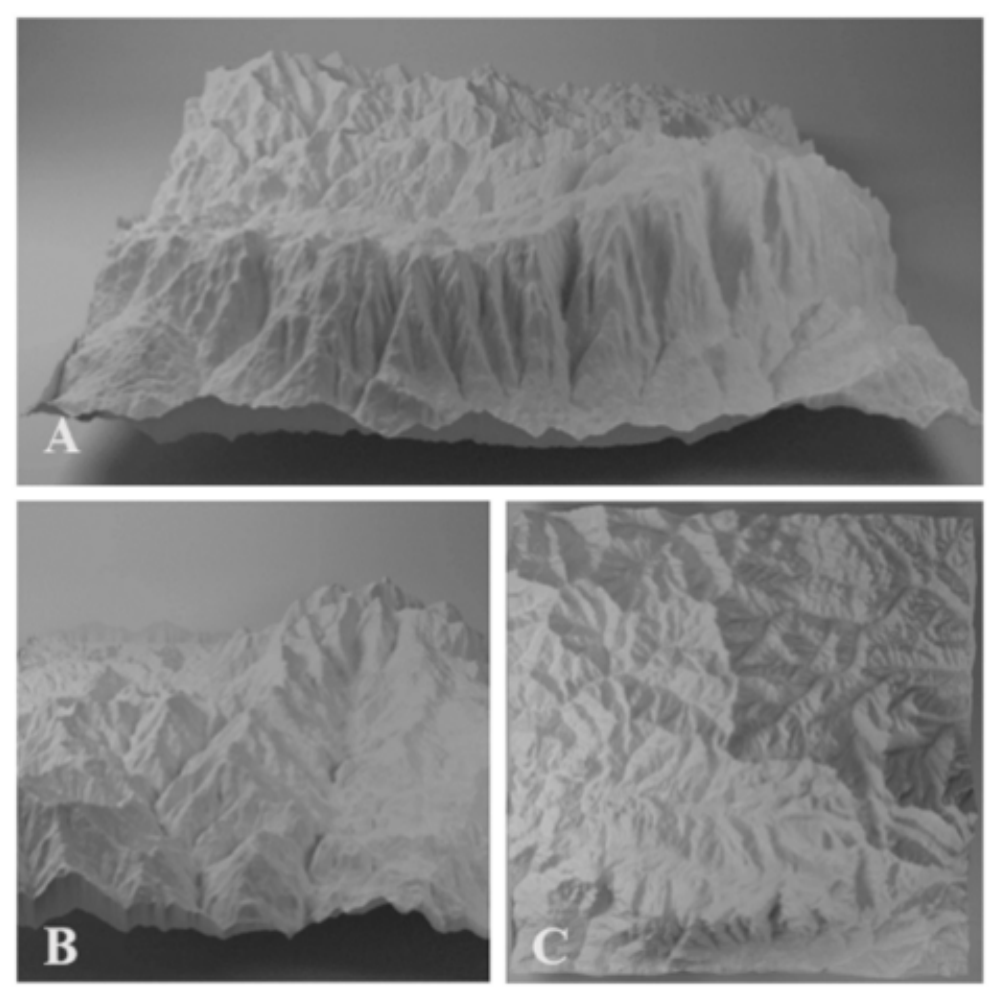

Fig. 4. Resulted 3D mesh: A. View from East, B. View from North, C. Top view 


\section{E. GPS and BARO Altitude Data}

The GPS data provides real-time positioning of the sub-ject, by recording the GPS coordinates and the BARO alti-tude data a relative accurate positon in a tridimensional space can be obtained.

\section{F. Spatial Placement of the 3D Model}

The DEM data is selected using a square area, which is defined by 4 points represent by their latitude and longitude values. To correlate the model we used the open street maps api to download an XML file from their servers, which then we used as a domain for the GPS coordinates. The routes are created by selecting the recording points in the open-source app Viking.

After scaling the GPS data and the 3D model to an appropriate domain we placed them into the WebGL Canvas and represented the path in a tridimensional space.

The biomonitoring component provides real time information regarding the physiological signs of the subjected, acquired directly from the monitoring devices. The interface is comprised of multiple charts and graphs.

The oxygen saturation is represented using a gradient of colors applied directly to the GPS route that is plotted on the three dimensional model.

\section{G. API}

Our api is the bond the ties the application together, it provides information about the subject, weather and alti- tude. We developed our API using JSON objects and implemented our backend logic using Node.js. The system routing was integrated using the NGINX server for static assets (geographical maps, HTML styles).

\section{H. LSTM Model}

Our LSTM model was developed using Tensor Flow in python. We implemented this model for fast detection and recognition of blood oxygen patterns and dynamic ECG analysis (Figure 5).

The models time step is manually adjusted along with other parameters such as the weights and memory alloca-tion. The model is directly integrated within our platform, and allows for fast matching of a set number of patterns (Figure 6).

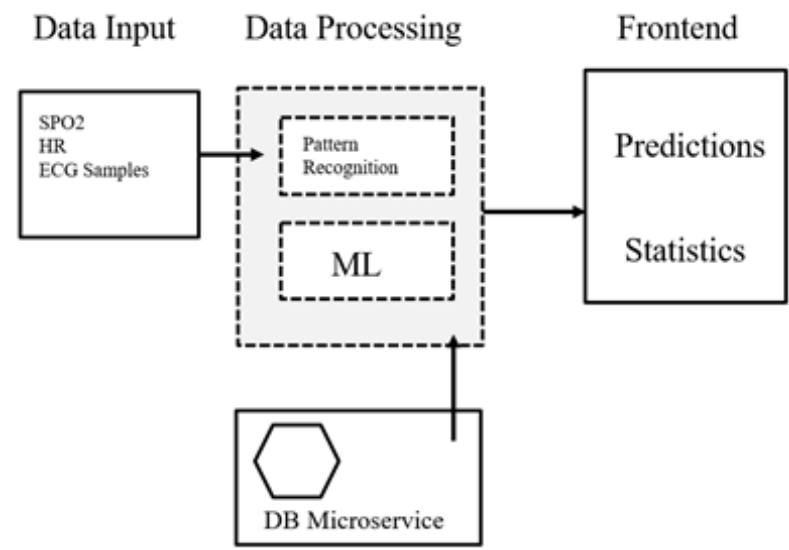

Fig. 5. Logical diagram of our services architecture

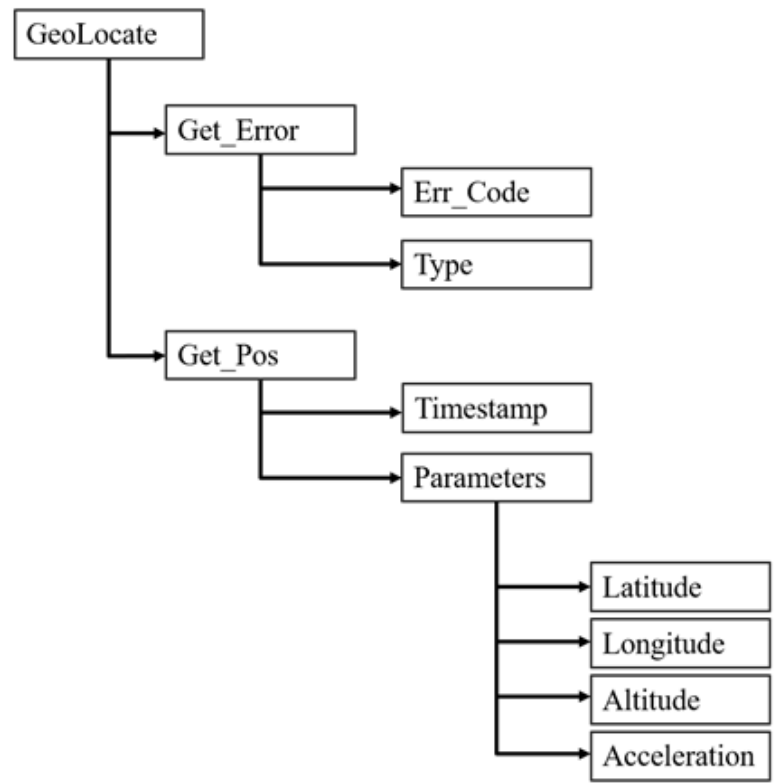

Fig. 6. The Three dimensional positioning API 


\section{CONCLUSION}

We presented a software platform devloped using scala-ble technologies such as linux containers and fast JavaScript APIs with JSON objects. Our LSTM model is implanted using python and is directly accessible during the monitoring sessions.

We believe our devops philosophy has ensured our platform for the foreseeable future. Our architecture allows for continuous integration of many more containers and many more computational models. While the satellite data provided a realistic representa-tion we believe aerial recordings could provide a more accu-rate data set for 3D topography.
The SRTM data and in some cases errors 20-30 meters and many types of artefacts resulted from the radar record-ings. Moreover, the mobile GPS and Barometric recordings are not very accurate, our application does not calculate a mean value or renders the whole recorded track but only a few selected points due to performance issues. An approxi-mation algorithm is required.

In the near future we will develop a new model that could profile and predict specific patterns of physical fit-ness.

\section{ACKNOWLEDGEMENT}

This paper was developed within the program National Project: GNaC 2018 ARUT no. 19/06.02.2018 from Politehnica University of Bucharest.

\section{REFERENCES}

[1] F. C. Adochiei, R. I. Ciucu, I. R. Adochiei, S. D. Grigorescu, G. C. Serițan, and M. Casian, “A web platform for rendring and viewing mri volumes using real-time raytracing principles," in 11th International Symposium on Advanced Topics in Electrical Engineering, Berlin, Germany, 2019.

[2] C. Miron, A. Păsărică, D. Arotăriței, H. Costin, R. G. Bozomitu, and C. otariu, "Hand gesture detection using a stereo camera system and simulation of movement,"' in 10th International Symposium on Advanced Topics in Electrical Engineering (ATEE), Bucharest, Romania, 2017. doi: https://doi.org/10.1109/ATEE.2017.7905134

[3] R. I. Ciucu, G. C. Seriţan, D. A. Dragomir, C. Cepişcă, and F. C. Adochiei, "ECG generation methods for testing and maintenance of cardiac monitors,' ' in E-Health and Bioengineering Conference, California, CA, 2015. doi: https://doi.org/10. 1109/EHB.2015.7391513

[4] H. Costin, "A fuzzy rules-based segmentation method for medical images analysis," International Journal of Computers Communications \& Control, vol. 8, no. 2, pp. 196-205, 2013. doi: https://doi.org/10.15837/ijccc.2013.2.301

[5] J. R. Santillan, M. Makinano-Santillan, and R. M. Makinano, "Vertical accuracy assessment of ALOS World 3D-30M digital elevation model over Northeastern Mindanao, Philippines," in IEEE International Geoscience and Remote Sensing Symposium, Tokyo, Japan, 2016.

[6] K. M. Attom, M. and N. Al-Akhras, "Geo environmental utilization of iron-filing with cement in soil stabilization," International Journal of Technology and Engineering Studies, vol. 2, no. 2, pp. 32-37, 2016. doi: https://doi.org/10.20469/ ijtes.2.40001-2

[7] C. L. S. Tablatin, F. F. Patacsil, and P. V. Cenas, "Design and development of an information technology fundamentals multimedia courseware for dynamic learning environment," Journal of Advances in Technology and Engineering Studies, vol. 2, no. 5, pp. 202-210, 2016. doi: https://doi.org/10.20474/jater-2.6.5

[8] B. Coustet, F. J. Lhuissier, R. Vincent, and J. P. Richalet, "Electrocardiographic changes during exercise in acute hypoxia and susceptibility to severe high-altitude illnesses," Circulation, vol. 131, no. 9, pp. 786-794, 2015. doi: https://doi. org/10.1161/CIRCULATIONAHA.114.013144

[9] C. Orphanidou and I. Drobnjak, "Quality assessment of ambulatory ECG using wavelet entropy of the HRV signal," IEEE Journal of Biomedical and Health Informatics, vol. 21, no. 5, pp. 1216-1223, 2016. doi: https://doi.org/10.1109/JBHI. 2016.2615316

[10] T. Cazangiu, F. Argatu, B. A. Enache, V. Vita, and S. Ganatsios, "Device for monitoring people with Alzheimer's disease," in International Symposium on Fundamentals of Electrical Engineering ISFEE, London, UK, 2018.

[11] T. Limgomonvilas, "Prediction for nonthaburi urban parks by integrated geo-informatics techniques," International Journal of Technology and Engineering Studies, vol. 3, no. 1, pp. 20-28, 2017. doi: https://doi.org/10.20469/ijtes.3. 40004-1

[12] R. Pan, D. Chua, J. S. Pathmasuntharam, and Y. P. Xu, "A WBAN based cableless ECG acquisition system," in 36th Annual International Conference of the IEEE Engineering in Medicine and Biology Society, Beijing, China, 2014. 
[13] C. M. Tung, "Vertical integration for smart manufacturing-the dynamic capability perspective," Journal of Advances in Technology and Engineering Studies, vol. 4, no. 2, pp. 70-78, 2018. doi: https://doi.org/10.20474/jater-4.2.3

[14] G. Forkuor and B. Maathuis, "Comparison of SRTM and ASTER derived digital elevation models over two regions in ghana-implications for hydrological and environmental modeling," in Studies on Environmental and Applied Geomorphology, New York, NY, 2012.

[15] H. Costin and C. Rotariu, "Medical image analysis and representation using a fuzzy and rule-based hybrid approach," International Journal of Computing Communication, vol. 1, pp. 156-162, 2006.

[16] C. Rotariu, A. Pasarica, G. Andruseac, H. Costin, and D. Nemescu, "Automatic analysis of the fetal heart rate variability and uterine contractions," in International Conference and Exposition on Electrical and Power Engineering (EPE), California, CA, 2014.

[17] K. Muralidharan, A. J. Khan, A. Misra, R. K. Balan, and S. Agarwal, "Barometric phone sensors: More hype than hope!" in Proceedings of the 15th Workshop on Mobile Computing Systems and Applications, Istanbul, Turkey, 2014, p. 12.

[18] R.-I. Ciucu, D.-A. Dragomir, I.-R. Adochiei, G.-C. Serițan, C. Cepişcă, and F.-C. Adochiei, “A non contact heart rate monitoring system for long-term assessments of HRV," in 10th International Symposium on Advanced Topics in Electrical Engineering (ATEE), New York, NY, 2017.

[19] C. Cepisca, F. C. Adochiei, S. Potlog, C. K. Banica, and G. C. Seritan, "Platform for bio-monitoring of vital parameters in critical infrastructures operation," in 7th International Conference on Electronics, Computers and Artificial Intelligence (ECAI), Berlin, Germany, 2015.

[20] G. G. Andruseac, A. Pasarica, C. O. Brezuleanu, G. Ignat, S. Brezuleanu, and C. L. Costuleanu, “An intelligent framework to manage and control an autonomous platform for detection, inspection and monitoring applications in chemical environments," Revista De Chimie, vol. 68, no. 6, pp. 1357-1360, 2017.

[21] R. Ciucu, F. Adochiei, I.-R. Adochiei, F. Argatu, G. Seriţan, B. Enache, S. Grigorescu, and V. V. Argatu, "Innovative devops for artificial intelligence," The Scientific Bulletin of Electrical Engineering Faculty, vol. 19, no. 1, pp. 58-63, 2019. doi: https://doi.org/10.1515/sbeef-2019-0011

[22] A. Păsărică, R. G. Bozomitu, D. Tărniceriu, G. Andruseac, H. Costin, and C. Rotariu, "Analysis of eye image segmentation used in eye tracking applications," Revue Roumaine Des Sciences Techniques-Serie Electrotech-nique Et Energetique, vol. 62, pp. 215-222, 2017.

[23] R. A. Drebin, L. Carpenter, and P. Hanrahan, "Volume ren dering," in Proceeding of 15th Annual Conference of Computing Graphic and Interactive Technonology, Dubai, UAE, 1988.

[24] C. Larco, R. Pahonie, and M. Mihaila-Andres, "Experimental study on mode I fracture of fibredux unidirectional prepeg," in AIP Conference Proceedings, Istanbul, Turkey, 2017. doi: https://doi.org/10.1063/1.4981977

[25] C. D. Correa and K.-L. Ma, "Visibility histograms and visibility-driven transfer functions," IEEE Transactions on Visualization and Computer Graphics, vol. 17, no. 2, pp. 192-204, 2010. doi: https://doi.org/10.1109/TVCG.2010.35

[26] J. Kniss, G. Kindlmann, and C. Hansen, "Multidimensional transfer functions for interactive volume rendering," IEEE Transactions on Visualization and Computer Graphics, vol. 8, no. 3, pp. 270-285, 2002. doi: https://doi.org/10.1109/ TVCG.2002.1021579

[27] B. D. Conner, S. S. Snibbe, K. P. Herndon, D. C. Robbins, R. C. Zeleznik, and A. Van Dam, "Three-dimensional widgets," in Proceedings of the 1992 Symposium on Interactive 3D Graphics, New Jersy, NJ. ACM, 1992. doi: https://doi.org/10. $1145 / 147156.147199$ 